\title{
Deterministic Equivalent for Max-Min SINR over Random User Locations
}

\author{
Hossein A.Moghaddam*, Antti Tölli*, Luca Sanguinetti ${ }^{\dagger \ddagger}$, Merouane Debbah ${ }^{\ddagger \S}$ \\ ${ }^{*}$ Center for wireless communications (CWC), University of Oulu, Oulu, Finland \\ ${ }^{\dagger}$ Dipartimento di Ingegneria dell'Informazione, University of Pisa, Pisa, Italy \\ ${ }^{\ddagger}$ Large Networks and System Group (LANEAS), CentraleSupélec, Université Paris-Saclay, Gif-sur-Yvette, France \\ $\S$ Mathematical and Algorithmic Sciences Lab, Huawei France, Paris, France
}

\begin{abstract}
The max-min signal-to-interference-plus-noise ratio (SINR) problem is considered in a coordinated network wherein $L$ base stations (BSs) each equipped with $N$ antennas serve in total $K$ single-antenna users that are uniformly distributed in the network. We conduct the analysis in the asymptotic regime in which $N$ and $K$ grow large to compute a deterministic approximation for the max-min SINR. The results are independent from fast-fading and users' locations and thus allow one to determine the optimal max-min SINR given basic system parameters such as cell radius, $K, N$ and pathloss exponent. The provided framework can be utilized for analyzing the problem without the need to run system level simulations and for finding the optimal $N, K$, resource allocation and BS placement. Numerical results are used to validate the analytical results in a finite system regime and to evaluate the effects of system parameters on the system performance.
\end{abstract}

\section{INTRODUCTION}

Coordinated multi point transmission (CoMP) has been studied extensively in literature as an efficient method to handle inter-cell interference (ICI) [1]. Due to fast fading channels, the impact of different system parameters has been largely investigated via Monte Carlo simulations. However, the advent of large dimensional networks (as envisioned in $5 \mathrm{G}$ networks) have spurred renewed interest in random matrix theory (RMT) [2] for the analysis and design of wireless communication systems. Even though the use of RMT allows one to remove the functional dependence of performance metrics from fast fading channels, it still requires a deterministic placement of user equipment terminals (UEs). In this work, we aim at computing asymptotic approximations of performance metrics that are independent of fading and UE locations. A similar approach is followed in [3] but for a simple one dimensional network in which only two base stations (BS) along with UEs are located on a line. An alternative approach to tackle such a problem is stochastic geometry as in [4] [6]. Despite being a very powerful tool, stochastic geometry has not yet led to tractable results considering advanced joint multi-cell processing [3].

Coordinated multi-cell resource allocation is generally formulated as an optimization problem in which a desired network utility is maximized subject to some requirements

This research was supported by the Academy of Finland (Decision no. 279101 and 284590). The research of L. Sanguinetti and M. Debbah has been supported by the ERC Starting Grant 305123 MORE.
[1], [7]. In this work, we consider the max-min signal-tointerference-and-noise ratio (SINR) problem, or equivalently, SINR balancing problem subject to a given total power constraint. This problem is closely related to the total power minimization problem subject to a given minimum SINR constraints. In [8], the authors proved that for multiple-inputsingle-output (MISO) downlink systems the two optimization problems are dual of each other. Both problems have been addressed extensively in the literature (see for example [1], [7], [9]-[13]). The max-min SINR problem was first addressed in [14] using an extended coupling matrix approach. The works in [8], [15] reformulate the problem based on conic programming. An approach based on nonlinear Perron-Frobenius theory [16] is proposed in [17] where the extension to MIMO downlink transmissions is given in [18]. The work in [19] considers the common rate maximization (or rate balancing) problem for the multi-antenna receiver case with per-antenna power constraint.

One of the first works considering large system analysis for linear receiver design is given by [20]. A single-cell setting under the assumption of Rayleigh fading is considered in [21], [22] whereas the multi-cell case is investigated in [23][25]. Under a more restrictive assumption, the authors in [26] provide an asymptotic analysis in a simple case where UEs in a network with two cells are required to be served with the same target rate. The analysis is extended in [27] for the minimization of the maximum power problem with homogeneous channel setting. The works in [28], [29] study the sum rate performance of zero-forcing (ZF) and regularized ZF precoding in large MISO broadcast systems. The work in [30] studies joint beamforming and power control in a coordinated multi-cell downlink system employing the nonlinear PerronFrobenius theory and RMT. The recent work in [31] focuses on the downlink and uplink of large-scale single-cell multiuser MIMO systems and aim at reducing the complexity of the linear SINR balancing precoder.

In this work, we utilize RMT to analyze multi-cell cooperative systems that account for channel fading and random UE locations. In particular, we do not assume a deterministic placement of user terminals but we average over random UE locations. As mentioned before, the max-min SINR and power minimization problems are closely related. The max-min SINR problem can be solved using a bisection method [32] where at

(C) 2015 IEEE. Personal use of this material is permitted. Permission from IEEE must be obtained for all other uses, in any current or future media, including reprinting/republishing this material for advertising or promotional purposes, creating new collective works, for resale or redistribution to servers or lists, or reuse of any copyrighted component of this work in other works. 
each iteration an instance of the power minimization problem is solved. Keeping this in mind, we first derive an asymptotic approximation for the total power consumption (given a set of SINR targets) as a function of SINR targets and basic system parameters such pathloss exponent, number of UEs and antennas and cell radios. Interpreting the total power consumption as power budget, we then get a relation between users' SINRs and basic system parameters which allows to determine the optimal SINR assignment. In the single-cell case, the solution to the max-min SINR problem is computed in closed form. For the general multi-cell case, the optimal SINR assignment is determined by using a bisection method. Note that the problem solved at each iteration is much simpler as compared to available methods that rely on convex optimization tools. Moreover, the resulting SINRs need to be recomputed only when the basic system parameters change. The results allow one to find approximations of the average system performance irrespective of fading and UE locations and to answer questions related to power budgeting, BS deployment strategies and network dimensioning. Moreover, the developed framework allows to study the aforementioned problems without the need to run Monte Carlo simulations.

The paper is organized as follows. The problem formulation is presented in Section II. The asymptotic analysis is provided in Section III and it is validated by means of numerical results in Section IV.

\section{System Model AND PROBlem Formulation}

Consider the downlink of a multi-cell multi-user MIMO system composed of $L$ cells where each BS has $N$ antennas. A total number of $K$ single-antenna UEs are randomly dropped in the coverage area. We assume that each UE is assigned to a single BS while being interfered by the other BSs. We denote the set of $K_{b}$ UEs served by BS $b$ as $\mathcal{U}_{b}$ and the index of the BS associated to UE $k$ as $b_{k}$. The set of all UEs is represented by $\mathcal{U}$ whereas $\mathcal{B}$ collects all BS indexes. Under this convention, we define $\mathbf{h}_{b, k} \in \mathbb{C}^{N}$ as the channel from BS $b$ to UE $k$ assuming that $\mathbf{h}_{b, k}=a_{b, k}^{1 / 2} \mathbf{z}_{b, k}$ where $\mathbf{z}_{b, k} \sim \mathcal{C N}\left(\mathbf{0}, \mathbf{I}_{N}\right)$ represents the small-scale fading with $a_{b, k}^{2}$ representing the large scale channel gain (or pathloss) from BS $b$ to UE $k$. Denoting by $\mathbf{v}_{k} \in \mathbb{C}^{N}$ and $p_{k}$ the normalized precoding vector and transmit power of UE $k$ from its intended $\mathrm{BS} b_{k}$, respectively, the received SINR at user $k$ is given by ${ }^{1}$ :

$$
{\tilde{\gamma_{k}}}^{\mathrm{DL}}=\frac{p_{k}\left|\mathbf{h}_{b_{k}, k}^{\mathrm{H}} \mathbf{v}_{k}\right|^{2}}{\sum_{j \in \mathcal{U}_{b} \backslash k} p_{j}\left|\mathbf{h}_{b, k}^{\mathrm{H}} \mathbf{v}_{j}\right|^{2}+\sigma^{2}}, \forall k \in \mathcal{U} .
$$

Under the assumption of Gaussian inputs with zero mean and unit variance, the max-min SINR problem can be formulated as [14]:

\footnotetext{
${ }^{1}$ The objective can be straightforwardly generalized to a weighted minimum SINR maximization where the SINR values are weighted by arbitrary user specific priority factors.
}

$$
\begin{aligned}
& \max _{\left\{\mathbf{v}_{k}, \mathbf{p}\right\}} \min _{k \in \mathcal{U}} \tilde{\gamma}_{k}^{\mathrm{DL}} \\
& \text { s.t. } \quad\|\mathbf{p}\|_{1} \leq P_{\mathrm{TX}} \\
&\left\|\mathbf{v}_{k}\right\|=1, \forall k \in \mathcal{U}
\end{aligned}
$$

The vector $\{\mathbf{p}\}$ contains $\left\{p_{k} \forall k \in \mathcal{U}\right\}$ values and the power available at BSs is upper bounded by a sum power constraint $\left\{P_{\mathrm{TX}}\right\}$. The thermal noise power $\sigma^{2}$ is normalized to unity throughout the work without loss of generality.

We now review the well-known iterative bisection solution for SINR balancing problem that is essentially based on the link between the SINR balancing and the power minimization under SINR constraints underlined in [1]. The idea behind the bisection algorithm is to solve a sequence of power minimization problems with fixed user specific SINR requirements imposed on the system at each step of the algorithm. While searching for the optimal SINR assignments, the bisection algorithm is stopped when the optimized sum power is within an $\epsilon$-distance from the power budget $P_{\mathrm{TX}}$.

The power minimization problem that need to be solved at each bisection iteration is given as [1]

$$
\begin{aligned}
\min _{\left\{\mathbf{p}, \mathbf{v}_{k}\right\}} & \sum_{b \in \mathcal{B}} \sum_{k \in \mathcal{U}_{b}} p_{k} \\
\text { s.t. } & \tilde{\gamma}_{k}^{\mathrm{DL}} \geq \gamma_{k}, \forall k \in \mathcal{U}
\end{aligned}
$$

where $\gamma_{k}$ is a fixed SINR target set by the outer bisection algorithm. The above problem can be solved using different approaches based on iterative uplink-downlink duality [1], on the centralized SOCP formulation [7] or using decentralized dual or primal decomposition techniques [7], [13]. The solution of interest in this work is uplink-downlink duality approach where the dual problem for (3) is formulated as [1]

$$
\begin{aligned}
\min _{\left\{\mathbf{v}_{k}\right\},\left\{\lambda_{k}\right\}} & \sum_{b \in \mathcal{B}} \sum_{k \in \mathcal{U}_{b}} \frac{\lambda_{k}}{N} \sigma^{2} \\
\text { s.t. } & \tilde{\gamma}_{k}^{\mathrm{UL}} \geq \gamma_{k}, \forall k \in \mathcal{U}
\end{aligned}
$$

where $\tilde{\gamma}_{k}^{\mathrm{UL}}$ is the dual UL SINR expression, and $\left\{\lambda_{k} / N\right\}$ correspond to the Lagrange multipliers associated to the SINR constraints in (3) and can be thought as the solutions to the above dual UL power minimization problem. ${ }^{2}$ An immediate consequence from duality is that under the given constraints both UL and DL have the same total power consumption. Moreover, the SINR targets can be achieved by the same set of beamforming vectors.

The solution to uplink problem is known to be given using a fixed point iteration formula as [1]

$$
\lambda_{k}(t+1)=\lambda_{k}(t) \frac{\gamma_{k}}{\tilde{\gamma}_{k}^{\mathrm{UL}}(t)}
$$

\footnotetext{
${ }^{2}$ Note that the Lagrange multipliers $\lambda_{k}$ are scaled by the factor $N$ to ensure that the total power of system is finite, when $N$ grows large.
} 
where $t$ is the iteration index and $\tilde{\gamma}_{k}^{\mathrm{UL}}(t)$ is given by

$\tilde{\gamma}_{k}^{\mathrm{UL}}(t)=\lambda_{k}(t) \mathbf{h}_{b_{k}, k}^{\mathrm{H}}\left(\sum_{j \in \mathcal{U} \backslash k} \lambda_{j}(t) \mathbf{h}_{b_{k}, j} \mathbf{h}_{b_{k}, j}^{\mathrm{H}}+N \mathbf{I}_{N}\right)^{-1} \mathbf{h}_{b_{k}, k}$

which corresponds to the SINR at iteration $t$ for given fixed uplink powers $\left\{\lambda_{k}(t) / N\right\}$. The optimal $\left\{\mathbf{v}_{k}\right\}$ correspond to a set of normalized minimum mean square error (MMSE) receivers:

$$
\mathbf{v}_{k}=\frac{\tilde{\mathbf{v}}_{k}}{\left\|\tilde{\mathbf{v}}_{k}\right\|}, \quad \tilde{\mathbf{v}}_{k}=\left(\sum_{j \in \mathcal{U} \backslash k} \lambda_{j} \mathbf{h}_{b_{k}, j} \mathbf{h}_{b_{k}, j}^{\mathrm{H}}+N \mathbf{I}_{N}\right)^{-1} \mathbf{h}_{b_{k}, k} .
$$

The SINR formulation in (6) tends to a deterministic value while system dimensions ( $N$ and $K$ ) grow large which in turn allows a deterministic expression for uplink powers in (5). In doing so, we perform large system analysis in the next section to get an accurate approximation for the solutions of the problems in hand.

\section{LARGE SYSTEM ANALYSIS}

We exploit the theory of large system analysis to compute first the so-called deterministic equivalents of uplink powers in the minimum power beamforming problem. We then utilize these results to find approximations of the optimal SINR assignment in the finite system regime. In doing so, we assume the following grow rate of the system dimensions:

Assumption 1. As $N \rightarrow \infty, 0<\liminf \frac{K}{N} \leq \limsup \frac{K}{N}<$ $\infty$.

Theorem 1. Under Assumptions 1, the power terms in (4) converge asymptotically to $\bar{\lambda}_{k}$ as $\lambda_{k}-\bar{\lambda}_{k} \rightarrow 0$ almost surely

$$
\bar{\lambda}_{k}=\frac{1}{\eta_{b_{k}}} \frac{\gamma_{k}}{a_{b_{k}, k}^{2}}
$$

and

$$
\eta_{b_{k}}=\left(1-\frac{1}{N} \sum_{j \in \mathcal{U}} \frac{1}{1+\frac{1}{\gamma_{j}} \frac{\eta_{b_{j}}}{\eta_{b_{k}}} \frac{a_{b_{j}, j}^{2}}{a_{b_{k}, j}^{2}}}\right)
$$

Proof: We formally prove $\lambda_{k}-\bar{\lambda}_{k} \stackrel{N \rightarrow \infty}{\longrightarrow} 0$ via a contradiction argument as in [25], [33] where a sketch of the proof is presented in Appendix A.

The above results give the deterministic equivalents for uplink powers irrespective of channel fading. However, the results still depend on users' locations. Thus, we proceed further by taking advantage of randomness of large scale pathgains. Based on these results, we first give the corresponding deterministic equivalent for $\eta_{b}$ in concise form irrespective of fading and users' locations. To this end, we make the following assumption on the network setting:

Assumption 2. Consider a honeycomb-structured cellular network with Rayleigh fading channel and UEs distributed within the cell radius $R$ of each cell. Assume also that the target SINRs $\left\{\gamma_{k}\right\}$ are taken from $0 \leq \gamma_{\min } \leq \gamma_{k} \leq \gamma_{\max }<\infty$ with a given probability distribution.

Then, using the Fubini theorem [2], an asymptotic approximation of $\eta_{b_{k}}$ in (9) can be computed as follows:

Lemma 1. If Assumptions $1-2$ hold true, then $\eta_{b}-\bar{\eta} \rightarrow 0$ almost surely with

$$
\bar{\eta}=1-\frac{K_{b}}{N}\left(\vartheta_{1}+\vartheta_{2}\right)
$$

where

$$
\begin{aligned}
& \vartheta_{1}=\int_{\gamma_{\min }}^{\gamma_{\max }} \frac{\gamma}{1+\gamma} F_{\gamma}(\gamma) d \gamma \\
& \vartheta_{2}=\sum_{b^{\prime} \neq b} \int_{-\pi}^{\pi} \int_{\gamma_{\min }}^{\gamma_{\max }} \int_{1}^{R} \frac{1}{1+\frac{a^{2}(r)}{\gamma a^{\prime 2}\left(\theta, r^{\prime}\right)}} F_{\theta}(\theta) F_{r}(r) F_{\gamma}(\gamma) d r d \gamma d \theta
\end{aligned}
$$

with $F_{x}(x)$ denoting the probability distribution function of random variable $x$. The number of users per cell $K_{b}$ is assumed to be the same for all BSs. Also, we have $r^{\prime}=$ $\sqrt{r^{2}+4 d_{b^{\prime}}^{2}+4 r d_{b^{\prime}} \cos \theta}$, where, $d_{b^{\prime}}$ denotes the distance between BSs $b^{\prime}$ and $b$.

Proof: The proof is given in appendix B.

Under uniform user distribution and SINR assignment, we simply have $F_{\gamma}(\gamma)=\frac{1}{\gamma_{\max }-\gamma_{\min }}, F_{\theta}(\theta)=\frac{1}{2 \pi}$ and $F_{r}(r)=\frac{1}{R}$. However, other settings are also possible by plugging corresponding probability distribution functions into the lemma. The quantity $\bar{\eta}$ in Lemma 1 can be plugged into (8) to obtain a deterministic equivalent for $\bar{\lambda}_{k}$ that depends only on its own path gain value. Given the pathloss dependent uplink powers $\bar{\lambda}_{k} / N$, we can derive the deterministic approximation for total power consumption as in the following corollary.

Corollary 1. If Assumptions $1-2$ hold true and $a_{b_{k}, k}$ is modeled as $a_{b_{k}, k}^{2}=\frac{1}{r^{\rho}}$ with $\rho$ being the pathloss exponent, the optimal total transmit power for serving $K=L K_{b} U E s$ in the network, under uniform user distribution and SINR assignment, can be approximated by

$$
P_{t}=\frac{L}{\bar{\eta}} \frac{K_{b}}{N} \chi=\frac{L}{1-\frac{K_{b}}{N}\left(\vartheta_{1}+\vartheta_{2}\right)} \frac{K_{b}}{N} \chi
$$

where $\chi=\frac{\gamma_{\max }^{2}-\gamma_{\min }^{2}}{2\left(\gamma_{\max }-\gamma_{\min }\right)} \frac{R^{\rho+1}-1}{R(\rho+1)} \sigma^{2}$.

Proof: The total power consumption is simply given by $P_{t}=\sum_{k} \frac{\lambda_{k}}{N}$ that converges to an expected value (due to strong law of large numbers) given by integration of (8) with respect to $r$ and $\gamma$, when $a_{b_{k}, k}^{2}=\frac{1}{r^{\rho}}$ and $\bar{\eta}$ is plugged into (8), and when $F_{\gamma}(\gamma)=\frac{1}{\gamma_{\max }-\gamma_{\min }}$ and $F_{r}(r)=\frac{1}{R}$.

Now, we have an approximation for total power consumption in asymptotic regime that is given as a function of SINR constraints and basic system parameters. In general, it is difficult to inversely present the SINRs as a function of total power. However, under a simplified single cell setting with $\gamma_{k}=\gamma_{\min }=\gamma_{\max }=\gamma$, we have the optimal SINRs in closed form. 
Corollary 2. If Assumptions $1-2$ hold true and $a_{b_{k}, k}$ is modeled as $a_{b_{k}, k}^{2}=\frac{1}{r^{\rho}}$ with $\rho$ being the pathloss exponent, the optimal SINR assignment $\gamma$ in a single cell setting is approximated as

$$
\begin{aligned}
& \gamma=\frac{-b+\sqrt{b^{2}+4 a}}{2 a} \\
& a=\frac{K_{b}}{N} \frac{R^{\rho+1}-1}{R(\rho+1)} \frac{\sigma^{2}}{P_{T X}}, b=a+\frac{K_{b}}{N}-1 .
\end{aligned}
$$

Proof: Assigning $F_{\gamma}(\gamma)=\delta(\gamma)$, where $\delta(\gamma)$ denotes the Dirac delta function, allows a closed form expression for integration in (11) which in turn gives an equation based on (12) whose solution results the optimal SINR assignment.

In the generic multi-cell setting, we can search for the optimal SINR assignment using bisection method. We define the problem as the search for the range of supported SINRs $\left[\gamma_{\min }, \gamma_{\mathrm{max}}\right]$ with a given power budget $P_{\mathrm{TX}}$. The feasibility of the problem for a given range can be easily checked using (12). Let us first define an SINR range parameter as $\gamma_{\text {range. }}$ In the bisection algorithm detailed below, we look for the maximum feasible SINR values $\gamma_{\max }$ with a given range $\gamma_{\text {range. }}$ The proposed method is presented in Algorithm 1.

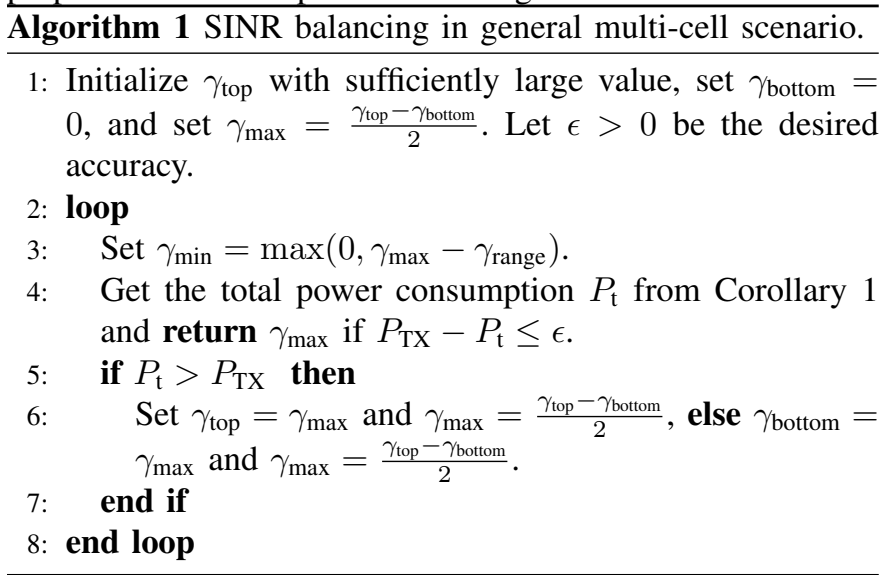

Compared to available methods in literature, this algorithm is particularly simple as it does not require solving any optimization problem. Instead, only a single equation is evaluated at each iteration. Moreover, the resulting SINRs are independent of fading and user locations and need to be computed only once for a given set of system parameters.

To conclude this section we present a feasibility condition as the final result that is determined by parameter $\eta$. Notice that $\eta$ in (10) and consequently power is positive and finite provided that $\frac{K_{b}}{N}\left(\vartheta_{1}+\vartheta_{2}\right)<1$. Therefore, a set of SINR targets can be satisfied depending on the sign of $\eta$ value. Later in numerical results, we investigate the effects of system parameters on the optimal SINR assignment and feasibility condition.

\section{NUMERICAL ANALYSIS}

We consider a network with $L=7$ cells and assume that UEs are equally distributed among cells. The pathloss function is modeled as $a_{b, k}=\left(d_{0} / d_{b, k}\right)^{2.5}$ where $\left\{d_{b, k}\right\}$ represents the distance between BS $b$ and UE $k$ and $d_{0}=1 \mathrm{~m}$ is the reference

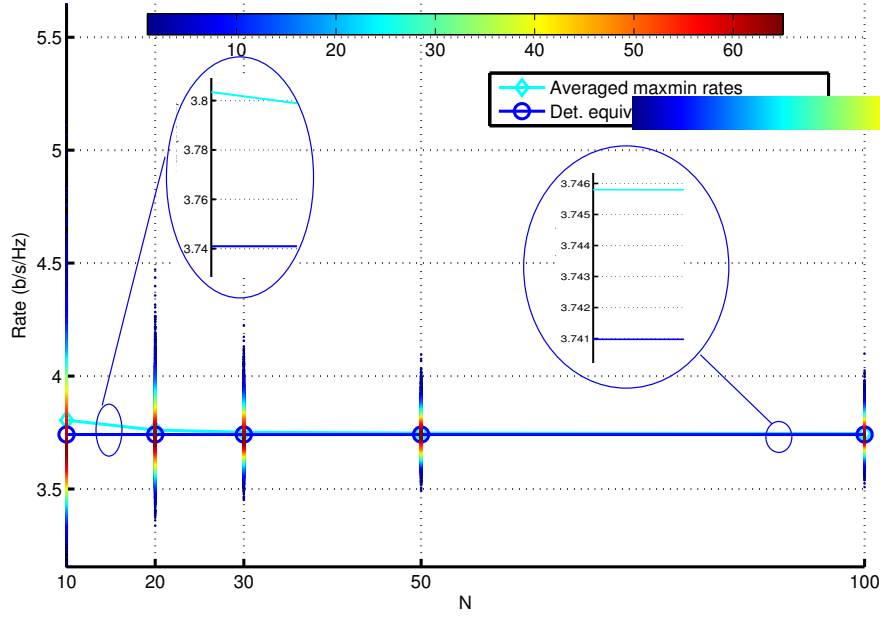

Fig. 1: The rate $(\mathrm{b} / \mathrm{s} / \mathrm{Hz})$ versus the number of antennas in a single cell scenario with $\mathrm{N} / \mathrm{K}=1, P_{\mathrm{TX}}=10 \mathrm{~mW}, \rho=2.5$ and $R=500 \mathrm{~m}$.

distance. The BSs are placed $1000 \mathrm{~m}$ apart from each other. The transmission bandwidth is $W=10 \mathrm{MHz}$ and the total noise power $\sigma^{2}=W N_{0}$ is $-104 \mathrm{dBm}$.

In order to evaluate the accuracy of the closed form SINR approximation in Corollary 2, a single cell setting is considered where users are dropped randomly on the coverage area and assigned with the same SINR target $\left(\gamma_{\max }=\gamma_{\min }\right)$ to be maximized given the power budget $P_{\mathrm{TX}}=10 \mathrm{~mW}$. For each random drop, uplink powers are calculated from Theorem 1, and the resulting sum power $\sum_{k} \bar{\lambda}_{k} / N$ is used in the bisection search to find the corresponding max-min SINR $\gamma$ (and the rate calculated as $\log _{2}(1+\gamma)$ ) values. The drop specific SINR (rate) values are then averaged over 1000 drops.

Fig. 1 shows the convergence of the averaged max-min rate to the deterministic equivalent given by (13) while system dimensions (total number of users $K$ and number of antennas per BS $N$ ) increase. The small gap in the case with $N=10$ indicates the fast convergence rate of the deterministic equivalents. The color bars depict the density of actual max-min rates for random users' drops. It can be seen that the drop specific max-min rates are mainly focused around the deterministic equivalents. Moreover, it can be observed that the variance of actual max-min rates is decreased while the dimensions are increased. Basically the same behavior can be observed also in the multi-cell scenario depicted in Fig. 2. In this case, the deterministic equivalents for max-min rates are found using bisection method (Alg. 1) with relatively high spatial loading ratio $K / N=4$.

Next, the effect of system parameters on the deterministic equivalents for max-min rates in multi-cell scenario is investigated. All users are assigned to be served with the same max-min rate $\left(\gamma_{\max }=\gamma_{\min }\right)$ while the total power budget is varied from $10 \mathrm{~mW}$ up to $5 \mathrm{~W}$ for different values of path-loss exponent $\rho$. Table I shows the resulted rates. The system parameters dictate the rates via the terms $\left(\vartheta_{1}+\vartheta_{2}\right)$ and $\chi$ in (12), where $\left(\vartheta_{1}+\vartheta_{2}\right)$ accounts for the effect of 


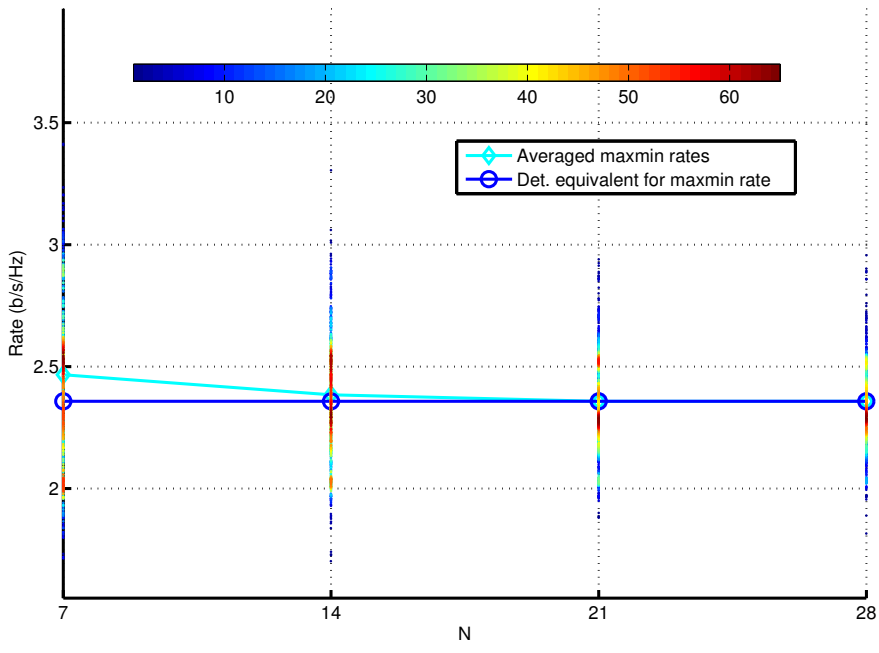

Fig. 2: The rate $(\mathrm{b} / \mathrm{s} / \mathrm{Hz})$ versus the number of antennas in a multi-cell scenario with $\mathrm{K} / \mathrm{N}=4, P_{\mathrm{TX}}=1 \mathrm{~W}, \rho=2.5$ and $R=$ $500 \mathrm{~m}$.

TABLE I: Maxmin rate vs. power budget and pathloss exponent, $\mathrm{R}=500 \mathrm{~m}$

\begin{tabular}{|c|c|c|c|c|}
\hline Pbud & $10 \mathrm{~mW}$ & $100 \mathrm{~mW}$ & $1 \mathrm{~W}$ & $5 \mathrm{~W}$ \\
\hline$\rho=2.5$ & 2.4 & 2.69 & 2.726 & 2.73 \\
\hline$\rho=3.5$ & 0.14 & 0.85 & 2.32 & 3.24 \\
\hline$\rho=4$ & 0.014 & 0.14 & 0.54 & 1.37 \\
\hline & \multicolumn{4}{|c|}{$\mathrm{b} / \mathrm{s} / \mathrm{Hz} / \mathrm{UE})$} \\
\hline
\end{tabular}

interference while $\chi$ depicts the signal energy attenuation. In general, increasing pathloss exponent decreases the supported rates as depicted in Table I. As an exception, in the case with $P_{\mathrm{TX}}=5 \mathrm{~W}$, a rise in pathloss exponent from 2.5 up to 3.5 results in an increase in the rate. This is due to the dominance of inter-cell interference where larger pathloss reduces the level of interference and results in a higher max-min rate. Another observation is the saturation of the supported maxmin rate as the power budget is increased. In the case with $\rho=2.5$, for example, increasing power budget beyond 100 $\mathrm{mW}$ improves the rate only marginally as the system becomes interference limited.

As mentioned before, the parameter $\eta$ gives a feasibility condition for a given set of system parameters. In order to study the effect of system parameters on the feasibility condition, we determine the required ratio of $K / N$ for a given target rate, pathloss exponent and cell radius directly from (12). As expected, Table II shows that a smaller ratio is required for satisfying increasing target rates. For very large target rates, every BS should have enough degrees of freedom (asymptotically one degree per user) to handle the increased inter-cell interference. This can be already observed in the table as the rate target is increased. However, higher spatial loading $K / N$ can be allowed for smaller target rates as the interference from more distant cells is reduced. Another observation is that the variation in cell radius does not alter the ratio $K / N$. This is simply due to the fact that the increasing cell size is compensated with a larger power budget for given
TABLE II: The feasibility condition versus system parameters

\begin{tabular}{|c|c|c|c|c|c|}
\multicolumn{1}{c}{ Target rate (b/s/Hz/UE) } & 1 & 2.6 & 3.4 & 4.4 & 5.7 \\
\hline $\mathrm{R}=100 \mathrm{~m}$ & 8.5 & 3.7 & 2.7 & 2.2 & 1.7 \\
\hline $\mathrm{R}=1000 \mathrm{~m}$ & 8.5 & 3.7 & 2.7 & 2.2 & 1.7 \\
\hline & \multicolumn{5}{|c|}{$\mathrm{K} / \mathrm{N}$} \\
\cline { 2 - 6 }
\end{tabular}

target rates.

\section{CONCLUSiOns}

Large system analysis of max-min SINR problem was considered in multi-cell multi-user network with coordinated beamforming. The results allow one to determine optimal max-min SINR assignment irrespective of users' locations and channel fading given basic system parameters such as cell radius, $K, N$ and pathloss exponent. The derived deterministic equivalents can be utilized for system dimensioning without the need to run system level simulations, i.e, finding the optimal $N, K$, resource allocation and BS placement.

\section{APPENDIX A}

\section{PRoOF OF THEOREM 1}

We begin with rewriting (5) as follows

$$
\frac{\gamma_{k}}{\lambda_{k}}=\frac{1}{N} \mathbf{h}_{b_{k}, k}^{\mathrm{H}}\left(\boldsymbol{\Sigma}_{b_{k}}^{\backslash k}+\mathbf{I}_{N}\right)^{-1} \mathbf{h}_{b_{k}, k}
$$

where $\boldsymbol{\Sigma}_{b_{k}}^{\backslash k}=\sum_{j \in \mathcal{U} \backslash k} \frac{\lambda_{j}}{N} \mathbf{h}_{b_{k}, j} \mathbf{h}_{b_{k}, j}^{\mathrm{H}}$. Due to dependency of variables $\left\{\lambda_{j}\right\}$ on channel vectors, it is not possible to apply trace lemma [2, Theorem 3.4] directly. Lets assume for a moment that $\left\{\lambda_{j}\right\}$ values are independent from channel vectors. Then, using trace lemma [2, Theorem 3.4] yields $\frac{1}{N} \mathbf{h}_{b_{k}, k}^{\mathrm{H}}\left(\boldsymbol{\Sigma}_{b_{k}}^{\backslash k}+\mathbf{I}_{N}\right)^{-1} \mathbf{h}_{b_{k}, k}-\frac{a_{b_{k}, k}^{2}}{N} \operatorname{Tr}\left\{\left(\boldsymbol{\Sigma}_{b_{k}}^{\backslash k}+\mathbf{I}_{N}\right)^{-1}\right\} \rightarrow$ 0 almost surely. Furthermore, applying rank-1 perturbation lemma [2, Theorem 3.9] and using (14) yields $\frac{\gamma_{k}}{\lambda_{k}}-$ $\frac{a_{b_{k}, k}^{2}}{N} \operatorname{Tr}\left\{\left(\boldsymbol{\Sigma}_{b_{k}}+\mathbf{I}_{N}\right)^{-1}\right\} \rightarrow 0$ almost surely. Finally, from well known random matrix theory results [2], we have $\frac{1}{N} \operatorname{Tr}\left\{\left(\boldsymbol{\Sigma}_{b_{k}}+\mathbf{I}_{N}\right)^{-1}\right\}-\eta_{b_{k}} \stackrel{N \rightarrow \infty}{\longrightarrow} 0$ almost surely, where $\eta_{b_{k}}$ is the Stieltjes transform of $\boldsymbol{\Sigma}_{b_{k}}$ and at the end from [28, Theorem 1] we get $\eta_{b}$ as in the corollary. We still need one more step to show the derived $\bar{\lambda}_{k}$ with independence assumption is a deterministic equivalent for $\lambda_{k}$ which can be proved via a contradiction argument as in [25], [33]

\section{APPENDIX B PROOF OF COROLlARY 1}

First we evaluate the error due to plugging $\frac{1}{1+\tau_{j}}$ instead of $\frac{1}{1+\tau_{j} \varsigma_{j}}$ in (9) as

$$
\frac{1}{1+\tau_{j}}-\frac{1}{1+\tau_{j} \varsigma_{j}}=\frac{\tau_{j}\left(\varsigma_{j}-1\right)}{\left(1+\tau_{j} \varsigma_{j}\right)\left(1+\tau_{j}\right)}=\iota_{j}
$$

where $\tau_{j} \triangleq \frac{a_{b_{j}, j}^{2}}{\gamma_{j} a_{b, j}^{2}}$ and $\varsigma_{j} \triangleq \eta_{b_{j}} / \eta_{b}$. Now, writing each term inside the summation of (9) as $\frac{1}{1+\tau_{j}}+\iota_{j}$, the error terms $\left\{\iota_{j}\right\}$ are averaged out by the summation, and hence, converge to $E\{\iota\}$ due to strong law of large number (SLLN). Bounding $|E\{\iota\}|$ using some inequalities, we get $|E\{\iota\}| \leq$ 


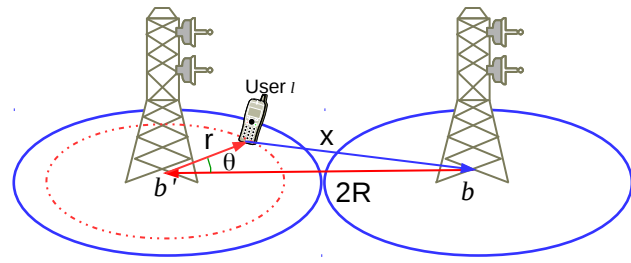

Fig. 3: Location of users in the network in polar coordinate

$|E\{\varsigma\}-1| \stackrel{(c)}{\approx} 0$. Finally, notice that $E\left\{\eta_{b}\right\}=\bar{\eta}, \forall b$ in the symmetric network defined in Assumption 2 which, using approximation $E\left\{\frac{\eta_{b}}{\eta_{b^{\prime}}}\right\} \approx \frac{E\left\{\eta_{b}\right\}}{E\left\{\eta_{b^{\prime}}\right\}}=\frac{\bar{\eta}}{\bar{\eta}}=1$ (see [34]), yields equality $(c)$. Thus, we can conclude that the error due to using $\frac{1}{1+\tau_{j}}$ instead of $\frac{1}{1+\tau_{j} \varsigma_{j}}$ in (9) disappears asymptotically.

Now, $\eta_{b}$ is given in a non-iterative form and we can proceed by averaging channel gain values. Figure 3 shows the position of a user in the serving BS with respect to neighbor cells. Since pathloss depends on distance between the user and the BS, we can average $\eta_{b}$ in (9) with respect to random entities $r$ and $\theta$, thanks to the strong law of large numbers.

\section{REFERENCES}

[1] H. Dahrouj and W. Yu, "Coordinated beamforming for the multicell multi-antenna wireless system," IEEE Transactions on Wireless Communications, vol. 9, no. 5, pp. 1748-1759, May 2010.

[2] R. Couillet and M. Debbah, Random Matrix Methods for Wireless Communications. Cambridge University Press, 2011.

[3] J. Hoydis, A. Müller, R. Couillet, and M. Debbah, "Analysis of multicell cooperation with random user locations via deterministic equivalents," in IEEE WiOpt, 2012.

[4] M. Haenggi, J. G. Andrews, F. Baccelli, O. Dousse, and M. Franceschetti, "Stochastic geometry and random graphs for the analysis and design of wireless networks," IEEE J. Sel. Areas Commun., vol. 27, no. 7, pp. 1029-1046, 2009.

[5] J. G. Andrews, F. Baccelli, and R. K. Ganti, "A tractable approach to coverage and rate in cellular networks," IEEE Transactions on Communications, vol. 59, no. 11, pp. 3122-3134, 2011.

[6] K. Huang and J. G. Andrews, "A stochastic-geometry approach to coverage in cellular networks with multi-cell cooperation," in Global Telecommunications Conference (GLOBECOM 2011), 2011 IEEE. IEEE, 2011, pp. $1-5$.

[7] A. Tölli, H. Pennanen, and P. Komulainen, "Decentralized minimum power multi-cell beamforming with limited backhaul signaling," IEEE Transactions on Wireless Communications, vol. 10, no. 2, pp. 570-580, Feb. 2011.

[8] A. Wiesel, Y. C. Eldar, and S. Shamai, "Linear precoding via conic optimization for fixed mimo receivers," IEEE Transactions on Signal Processing, vol. 54, no. 1, pp. 161-176, 2006.

[9] — - "Linear precoding via conic optimization for fixed MIMO receivers," IEEE Transactions on Signal Processing, vol. 54, no. 1, pp. 161-176, Jan 2006.

[10] E. Bjornson, M. Bengtsson, and B. Ottersten, "Optimal multiuser transmit beamforming: A difficult problem with a simple solution structure [lecture notes]," IEEE Signal Processing Magazine, vol. 31, no. 4, pp. 142-148, July 2014.

[11] H. Pennanen, A. Tölli, and M. Latva-Aho, "Decentralized coordinated downlink beamforming via primal decomposition," IEEE Signal Processing Letters, vol. 18, no. 11, pp. 647-650, 2011.

[12] C. Shen, T.-H. Chang, K.-Y. Wang, Z. Qiu, and C.-Y. Chi, "Distributed robust multicell coordinated beamforming with imperfect CSI: An ADMM approach," IEEE Transactions on Signal Processing, vol. 60, no. 6, pp. 2988-3003, 2012.
[13] H. Pennanen, A. Tölli, and M. Latva-aho, "Multi-cell beamforming with decentralized coordination in cognitive and cellular networks," IEEE Transactions on Signal Processing, vol. 62, no. 2, pp. 295-308, 2014.

[14] W. Yang and G. Xu, "Optimal downlink power assignment for smart antenna systems," in Proceedings of the 1998 IEEE International Conference on Acoustics, Speech and Signal Processing, 1998., vol. 6. IEEE, 1998, pp. 3337-3340.

[15] Y. Huang, G. Zheng, M. Bengtsson, K.-K. Wong, L. Yang, and B. Ottersten, "Distributed multicell beamforming with limited intercell coordination," IEEE Transactions on Signal Processing, vol. 59, no. 2, pp. $728-738,2011$.

[16] U. Krause, "Concave perron-frobenius theory and applications," Nonlinear Analysis: Theory, Methods \& Applications, vol. 47, no. 3, pp. 1457-1466, 2001.

[17] C. W. Tan, M. Chiang, and R. Srikant, "Maximizing sum rate and minimizing MSE on multiuser downlink: Optimality, fast algorithms and equivalence via max-min SINR," IEEE Transactions on Signal Processing, vol. 59, no. 12, pp. 6127-6143, Dec 2011.

[18] D. W. Cai, T. Q. Quek, and C. W. Tan, "A unified analysis of maxmin weighted SINR for mimo downlink system," IEEE Transactions on Signal Processing, vol. 59, no. 8, pp. 3850-3862, 2011.

[19] A. Tölli, M. Codreanu, and M. Juntti, "Linear multiuser MIMO transceiver design with quality of service and per-antenna power constraints," IEEE Transactions on Signal Processing, vol. 56, no. 7, pp. 3049-3055, July 2008.

[20] D. N. C. Tse and S. V. Hanly, "Linear multiuser receivers: Effective interference, effective bandwidth and user capacity," IEEE Transactions on Information Theory, vol. 45, no. 2, pp. 641-657, 1999.

[21] L. Sanguinetti, E. Björnson, M. Debbah, and A. L. Moustakas, "Optimal linear precoding in multi-user MIMO systems: A large system analysis," in 2014 IEEE Global Communications Conference. IEEE, 2014, pp. 3922-3927.

[22] L. Sanguinetti, A. L. Moustakas, E. Björnson, and M. Debbah, "Large system analysis of the energy consumption distribution in multi-user MIMO systems with mobility," IEEE Trans. Wireless Commun., vol. 14, no. 3, pp. 1730-1745, 2015.

[23] S. Lakshminaryana, J. Hoydis, M. Debbah, and M. Assaad, "Asymptotic analysis of distributed multi-cell beamforming," in 21 st IEEE PIMRC, 2010, pp. 2105-2110.

[24] S. Lakshminaryana, M. Assaad, and M. Debbah, "Coordinated multi-cell beamforming for massive MIMO: A random matrix approach," IEEE Transaction on Information Theory, vol. 61, no. 6, 2015.

[25] L. Sanguinetti, R. Couillet, and M. Debbah, "Large system analysis of base station cooperation for power minimization," IEEE Transactions on Wireless Communications, vol. 15, no. 8, pp. 5480-5496, Aug 2016.

[26] R. Zakhour and S. Hanly, "Base station cooperation on the downlink: Large system analysis," IEEE Trans. Inf. Theory, vol. 58, no. 4, pp. 2079-2106, Apr. 2012.

[27] R. Zakhour and S. V. Hanly, "Min-max power allocation in cellular networks with coordinated beamforming," IEEE Journal on Selected Areas in Communications, vol. 31, no. 2, pp. 287-302, 2013.

[28] S. Wagner, R. Couillet, M. Debbah, and D. Slock, "Large system analysis of linear precoding in correlated MISO broadcast channels under limited feedback," IEEE Transactions on Information Theory, vol. 58, no. 7, pp. 4509-4537, July 2012.

[29] J. Zhang, C.-K. Wen, S. Jin, X. Gao, and K.-K. Wong, "Large system analysis of cooperative multi-cell downlink transmission via regularized channel inversion with imperfect CSIT," IEEE Trans. Wireless Commun., vol. 12, no. 10, 2013.

[30] Y. Huang, C. W. Tan, and B. D. Rao, "Joint beamforming and power control in coordinated multicell: Max-min duality, effective network and large system transition," IEEE Transactions on Wireless Communications, vol. 12, no. 6, pp. 2730-2742, 2013.

[31] H. Sifaou, A. Kammoun, L. Sanguinetti, M. Debbah, and M. Alouini, "Max-min SINR in large-scale single-cell MU-MIMO: asymptotic analysis and low complexity transceivers," CoRR, vol. abs/1605.07480, 2016. [Online]. Available: http://arxiv.org/abs/1605.07480

[32] S. Boyd and L. Vandenberghe, Convex Optimization. Cambridge University Press, 2004.

[33] H. Asgharimoghaddam, A. Tölli, L. Sanguinetti, and M. Debbah, "Decentralized multi-cell beamforming with QoS guarantees via large system analysis," in IEEE CAMSAP, Dec 2015.

[34] S. A. O. K. Kendall, M., Kendall's Advanced Theory of Statistics, 6th ed. London: Wiley-IEEE Press, 1998. 